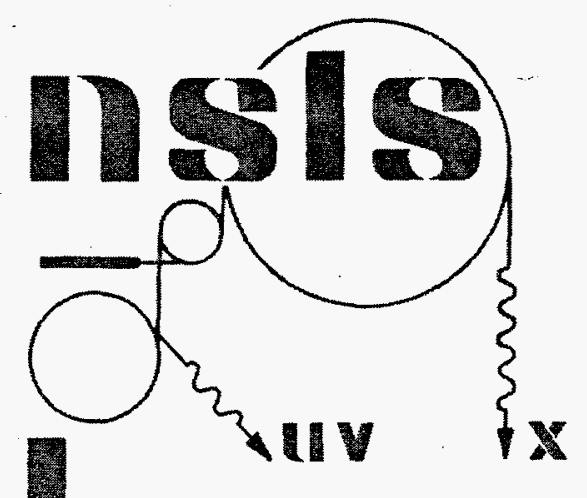

\title{
Data Acquisition and Analysis of Mammography Images at the NSLS June - August 1995
}

F. Arfelli, C. Burns, D. Chapman, N. Gmur, R. E. Johnston, R. Menk, E. Pisano, D. Sayers, W. Thomlinson, D. Washburn, Z. Zhong

\section{NATIONAL SYNCHROTRON LIGHT SOURCE}

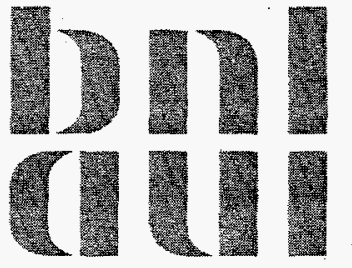

BROOKHAVEN NATIONAL LABORATORY ASSOCIATED UNIVERSITIES, INC.

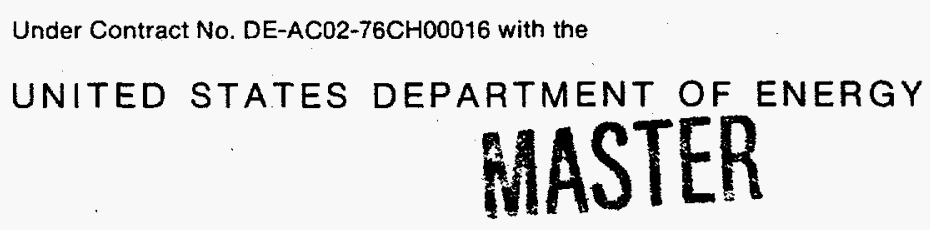

DISTRIDUTION OF THLS DOCUMENT IS UNLLMITED 


\section{DISCLAIMER}

This report was prepared as an account of work sponsored by an agency of the United States Government. Neither the United States Government nor any agency thereof, nor any of their employees, nor any of their contractors, subcontractors, or their employees, makes any warranty, express or implied, or assumes any legal liability or responsibility for the accuracy, completeness, or usefulness of any information, apparatus, product, or process disclosed, or represents that its use would not infringe privately owned rights. Reference herein to any specific commercial product, process, or service by trade name, trademark, manufacturer, or otherwise, does not necessarily constitute or imply its endorsement, recommendation, or favoring by the United States Government or any agency, contractor or subcontractor thereof. The views and opinions of authors expressed herein do not necessarily state or reflect those of the United States Government or any agency, contractor or subcontractor thereof. 


\section{DISCLAMMER}

Portions of this document may be illegible in electronic image products. Images are produced from the best available original document. 


\title{
Data Acquisition and Analysis of Mammography Images at the NSLS June - August 1995
}

\author{
F.Arfelli ${ }^{1}$, C.Burns ${ }^{2}$, D.Chapman ${ }^{1,4}$, N.Gmur ${ }^{1}$, R.E.Johnston ${ }^{2}$, R.Menk ${ }^{1}$, E.Pisano ${ }^{2}$, \\ D.Sayers ${ }^{3}$, W.Thomlinson', D.Washburn' ${ }^{2}$, Z.Zhong ${ }^{1}$
}

1. NSLS, Brookhaven National Laboratory, Upton, NY, 11973

2. Department of Radiology, University of North Carolina, Chapel Hill, N.C.27599

3. Department of Physics, North Carolina State University, Raleigh, N.C. 27695

4. Illinois Institute of Technology, Chicago, I1 60616

\section{Introduction}

The display of low-contrast tissue structures and the finest microcalcifications is essential for the early diagnosis of breast cancer $(1,2,3)$. From this requirement, it should be noted that the $\mathrm{x}$-ray imaging beam energy must be matched to the object thickness and the tissue composition of the breast in order to achieve high image quality with minimum radiation exposure to the patient (4-10). With the conventional molybdenum anode $\mathrm{x}$-ray tube, widely used for approximately the last 20 years, the possibilities of adapting the beam energy to the object thickness or the density of the breast tissue are severely limited since the relative energy distribution of the $\mathrm{x}$-ray spectrum changes very little by varying the $x$-ray tube voltage.

The synchrotron radiation $\mathrm{x}$-ray source provides a high flux over a wide continuous energy spectrum. This presents the possibility of obtaining a monochromatic tunable beam in order to choose the optimal energy that can increase the information content in the images or reduce the patient exposure. The intrinsic vertical collimation of the synchrotron beam may reduce the scattered radiation. Antiscattering grids used in the conventional mammographic system are not necessary. They may absorb more than $50 \%$ of the x-rays exiting from the breast, causing the dose to be more than doubled in order to maintain image quality.

At Brookhaven National Laboratory mammography experiments are being carried out at the X27C R\&D beamline of the National Synchrotron Light Source using a monochromatic $\mathrm{x}$-ray beam in order to explore the potential of monoenergetic photons for mammographic imaging. Preliminary reports of some of the work have been submitted for publication as conference proceedings $(11,12)$. Our measurements and results occurred during two separate one week periods of beam time; the first one in June and the second one in August 1995.

The monochromatic $x$-ray beam used for the imaging was produced by a double crystal Si(111) Bragg monochromator which can select energies from 15 to $25 \mathrm{keV}$. Conventional mammographic phantoms, including a contrast-detail phantom, the 
ACR (American College of Radiology) phantom and an in-vitro excised tissue sample, were imaged. The detector was a Fuji Image plate with a spatial resolution of 100 microns. Many images of the same phantoms were also recorded on a conventional mammographic film-screen system. The images were acquired in a line scan mode simultaneously moving the phantom and the detector through the beam. These images were compared with images produced using a conventional mammographic system at the University of North Carolina. The synchrotron images show a better contrast than the conventional images when compared to the theoretical contrast.

\section{Experimental set-up}

The experimental set-up is shown in Figure 1. The overall length of the system from the monochromator to the detector is 2.8 meters. The $85 \mathrm{~mm}$ wide white beam is provided by the X27C bending magnet source roughly 20 meters from the experimental hutch. The set-up, that is not under vacuum, begins downstream of a beryllium window. At that point a slit provides a $0.5 \mathrm{~mm}$ high beam which is incident on a double crystal $\mathrm{Si}(111)$ Bragg monochromator. The monochromator consists of two independent crystals $150 \mathrm{~mm}$ wide providing complete horizontal acceptance of the beam. The lengths of the first and second crystals are $60 \mathrm{~mm}$ and $90 \mathrm{~mm}$, respectively, chosen in order to allow a vertical acceptance up to $3 \mathrm{~mm}$ in the energy range from 15 to $25 \mathrm{keV}$ (corresponding to a Bragg angle range from 7.6 to 4.5 degrees). The energy resolution $\Delta \mathrm{E} / \mathrm{E}$ is $5 \times 10^{-4}$ at $18 \mathrm{keV}$. The monochromator aluminum chamber is kept in a He atmosphere. The first water-cooled crystal holder is attached to a Huber rotational stage which allows the Bragg angle to be set with an angular resolution of $2.5^{*} 10^{-4} \mathrm{deg}$. The beam diffracted by the first crystal has another Bragg reflection from the second crystal set in parallel configuration in order to keep the outgoing beam parallel to the incident beam but with an offset in the height which is about $20 \mathrm{~mm}$ (about twice the distance of $10 \mathrm{~mm}$ between the crystals). There is a slightly variation in the height when changing the Bragg angle but it is negligable for our purposes. The second crystal holder is fixed to the same frame as the first one in order to rotate the two crystals simultaneously. It is provided with fine adjustments by means of three remote-controlled micrometric screws moved by a piezo with a resolution of 0.1 micron (Picomotor driver, New Focus). With these adjustments a good alignment of the two crystals can be achieved. That is fundamental for maximizing the transmitted flux and obtaining a uniform beam over its width. The energy calibration of the monochromator is done by measuring the $\mathrm{k}$-absorption edges of Mo and Rh foils. Each time the working energy is changed the correct position of the second crystal is checked with a fine scan until the maximum flux transmission is reached. That means that a good overlap of the rocking curves of the two crystals exists.

At the exit of the monochromator the beam is collimated by lead shielding. Downstream of the monochromator there is a fast shutter with an ionization chamber in front of it which is used to monitor the strength of the incoming beam before opening the imaging shutter. Another slit is located downstream of the shutter, 
approximately $110 \mathrm{~cm}$ in front of the object to be imaged. The dose monitor ionization chamber is placed after this slit and is used to measure the entrance dose to the phantom (Section 4). The detector is placed about $100 \mathrm{~cm}$ downstream of the phantom with a final slit just in front of it to reduce the scattered radiation from the phantom on the image plate. The detector and phantom are fixed on the same support and are moved vertically by a Klinger stepper motor driver.

\section{Method}

The detector is a Fuji ST3 PSP image plate used with a Fuji BAS 2000 image plate reader. Typical reading parameters are: sensitivity of 4000 or 400 , latitude of 4 and a resolution size of 100 microns. The plates are read out as a $2048 \times 2560$ matrix (100 microns/pixel). Conventional mammographic film has also been used to record the images in conjunction with a Film Quick-CT film processor. Since the beam size is $0.5 \mathrm{~mm}$ high $\times 85 \mathrm{~mm}$ wide the images were acquired in line scan mode. The phantom and the detector, placed on the same vertical movement stage, were moved simultaneously through the beam by the stepper motor driver with a maximum speed of $16 \mathrm{~mm} / \mathrm{sec}$.

Data acquisition software has been developed to automate all the control and scan procedures. The input parameters required are: the beam energy, beam width, the phantom and filter thicknesses, the starting and final positions for the scan and the scan speed. The speed can be set directly or can be automatically chosen by the program for a predetermined detector exposure or, optionally, for a predetermined entrance dose to the phantom. The procedure reads the output current from the monitor ionization chamber (the one upstream of the fast shutter) and calculates the necessary speed. The current from the ionization chamber is amplified by a Keithley amplifier with the gain set at $10^{9}$ and the output voltage is converted into counts/sec by means of a voltageto-frequency converter which is connected to a scaler. Knowing the current I from the counts/sec, the relationship between the incident photon number/sec $N_{I}$ and the current I from the ionization chamber is the following:

$$
\mathrm{N}_{\mathrm{I}}=\frac{\mathrm{I} \omega_{\text {ion }}}{\operatorname{Ee}\left(1-\mathrm{e}^{-\mu_{m}^{\mathrm{gsd}}}\right)}
$$

where $\mathrm{E}$ is the beam energy, $\mathrm{e}$ is the electron charge, $\omega_{\text {ion }}$ is the ionization energy for the gas in the chamber (nitrogen at atmospheric pressure), $\mu_{e n}^{g a s}$ is the absorption coefficient of the gas and $d$ is the thickness of the ionization chamber.

The total photon number $\mathrm{N}_{\text {tot }}$ per $\mathrm{cm}^{2}$ for a translation length $\mathrm{h}$, a beam width $\mathrm{w}$ and a translation time $\Delta \mathrm{T}$ is 


$$
N_{\text {tot }}=\frac{N_{I} \Delta T}{w h}=\frac{N_{I}}{S w}
$$

where $\mathbf{s}$ is the scan speed. The requirements on detector exposure or phantom entrance dose are correlated to the incident photon number $\mathrm{N}_{\text {tot }}$ by means of the relationships shown in Section 4. In this way the scan speed is determined from a given $N_{\text {tot }}$. Thus, it is possible to increase or decrease the exposure by controlling the speed. There is, however, an intrinsic limit which is the maximum speed available and this means that the use of lucite and aluminum filters may be necessary directly after the monochromator.

When the scan starts, there is an acceleration phase arranged in such a way that the driver reaches the constant predetermined speed at the starting position at which time the shutter is opened. When the scan is at the end position the shutter is closed and the deceleration phase occurs. In practice, the total translation length is larger than the actual imaged phantom height to allow an imaging scan phase with constant speed and uniform exposure. For each run all the parameters used in the scan and the counts from the first and second ionization chambers are stored in a data file for off-line calculation.

The second ionization chamber (dose monitor chamber), placed after the fast shutter, measures the actual incident photon number on the phantom surface and from this measurement all the actual dose and exposure evaluations are performed, as described in Section 4. A scaler integrates the monitor chamber counts over the whole scan time corresponding to a total charge $Q_{\text {tot }}$ produced in the ionization chamber by the incident beam. The actual incident photons $/ \mathrm{cm}^{2} \mathrm{~N}_{\mathrm{inc}}$ is then given by:

$$
\mathrm{N}_{\mathrm{inc}}=\frac{1}{\mathrm{hW}} \frac{\mathrm{Q}_{\mathrm{tot}} \omega_{i o n}}{\operatorname{Ee}\left(1-\mathrm{e}^{-\mu_{n n} \mathrm{~d}}\right)}
$$

where the product $h^{*} w$ represents the imaged area.

\section{Dosimetry}

Although mammography is uniquely effective in early detection of breast cancer, breast tissue is sensitive to radiation carcinogenesis. Although the resulting benefits of this examination substantially exceed potential risks, the dose must be monitored and minimized. Five major variables affect the breast dose delivered in a mammographic examination: the choice of the imaging system, the $x$-ray beam energy ( $H L V)$, the degree of breast compression employed and the breast size and adiposity.

Various dose parameters that might be considered are the in-air exposure at the position of the entrance surface of the breast $\left(\mathrm{X}_{\mathrm{a}}\right)$, the dose to the entrance surface of the breast $\left(\mathrm{D}_{3}\right)$, the dose to the midline of the breast $\left(\mathrm{D}_{\text {mid }}\right)$, and the mean dose to the glandular tissue of the breast $\left(\mathrm{D}_{\mathrm{g}}\right)$. In-air surface exposure is easy to measure with an ionization chamber and from this measurement it is easy to determine the surface dose but it gives an overestimation of the cancer risk. Midline dose is difficult to measure 
directly (it can represent the risk to the glandular tissue) but it is an underestimation of the risk for the low-energy beams used for film-screen mammography. Mean glandular dose provides the best indicator of the potential carcinogenic radiation risk to the patient from mammographic examination. It commonly is assumed that the cancer risk is linearly related to the dose and that the breast cancers arise in the glandular tissue which is the most vulnerable when compared to adipose, skin and areolar tissues. Mean glandular dose cannot be measured directly but must be calculated from the result of simple measurements and tabulated values. The exposure as a function of the depth may be measured in phantoms using thermoluminescent dosimeters (TLD). Then the doses can be calculated from exposure levels using exposure-to-dose conversion factors.

Although the average glandular dose is the quantity usually reported, dose is sometimes given as the normalized average glandular dose $\left(\mathrm{D}_{\mathrm{gN}}\right)$, which is the dose $\left(D_{g}\right)$ normalized to the unit exposure in air at the entrance surface of the breast $\left(X_{a}\right)$. The value $X_{a}$ is set depending upon the detection system sensitivity in order to achieve a desired final image density. Once the exposure in air $X_{a}$ is measured, then it is multiplied by the values of $D_{g N}$ to arrive at reasonably accurate estimates of the average glandular dose $\left(\mathrm{D}_{\mathrm{g}}\right)$ to the patient. For conventional mammography the normalized dose $\mathrm{D}_{\mathrm{gN}}$ is determined by the HVL and breast thickness. Tables of values have been provided by a number of sources, including the National Council on Radiation Protection and Measurements (13) and the Center for Devices and Radiological Health (14).

A simplified model for the human breast used for dosimetry is proposed by Hammerstein et al. (15) to compute the average glandular dose in a firmly compressed breast with a rectangular cross section. The assumptions are that there is an outer layer of adipose tissue, not containing glandular tissue, that is roughly $0.5 \mathrm{~cm}$ thick on the outer (upper and lower) surfaces of the breast and there is a central portion of breast tissue composed of a uniform mixture of $50 \%$ adipose and $50 \%$ glandular tissue. Typical breast thickness after firm compression is $45 \mathrm{~mm}$, although thickness can vary in a range from $15 \mathrm{~mm}$ to $75 \mathrm{~mm}$.

In our mammographic experiments with synchrotron radiation it has been possible to implement a procedure for dose and exposure evaluations for each acquired image. We use simple relationships which consider the monoenergetic spectrum as well as the good geometric conditions due to the laminar beam and the slit system.

As shown above (Section 3), it is possible to determine the number $N_{0}$ of incident photons per surface unit (impinging on the phantom) from the output signal of the second ionization chamber, at a given energy $\mathrm{E}$. The procedure developed for the estimation of dose determines $\mathrm{N}_{0}\left(\mathrm{ph} / \mathrm{cm}^{2}\right)$, then the entrance dose to the lucite phantom is calculated, using the relationship:

$$
\mathrm{D}_{\mathrm{s}}=\mathrm{N}_{0} \mathrm{E} \frac{\mu_{e n}^{l u c}}{\rho^{l u c}}
$$

where $\mu_{e n}^{\text {luc }}$ is the absorption coefficient of the phantom and $\rho^{\text {luc }}$ is its density. In order to have a standard comparison of doses (since the thickness $t$ of lucite can be different 
at the different energies to allow a fixed exposure to the detector) the procedure calculates the entrance dose for a $42 \mathrm{~mm}$ thick lucite phantom, using $\mathrm{N}_{0}^{\prime}=\mathrm{N}_{0} \mathrm{e}^{-\mu_{h e}(\mathrm{t}-4.2)}$ instead of $\mathrm{N}_{0}$ as incident flux, where $\mu_{\text {tuc }}$ is the lucite attenuation coefficient.

The procedure also provides the exposure to the detector, $E_{D}$, using the relationship:

$$
\mathrm{D}_{\text {air }}(\mathrm{rad})=0.877 \mathrm{E}_{\mathrm{D}}(\mathrm{R})=\mathrm{N}_{\mathrm{D}} \mathrm{E} \frac{\mu_{e n}^{\text {air }}}{\rho^{\text {air }}}
$$

where $\mathrm{N}_{\mathrm{D}}=\mathrm{N}_{0} \mathrm{e}^{-\mu_{\text {hxt }}}$ is the photon number per surface unit reaching the detector.

Finally, the procedure calculates the average glandular dose that should be delivered to a breast of thickness $t$ in order to obtain an image corresponding to a given exposure to the detector. The breast thickness usually taken into consideration is $\mathrm{t}=45 \mathrm{~mm}$, which is standard, as well as $\mathrm{t}=20,40,42$, and $70 \mathrm{~mm}$.

Considering the standard breast composition described above and the breast thickness $t$, the photon number on the detector, $N_{D}$, for an image is related to the incident photon flux per unit surface area of the breast, $\mathrm{N}_{t}$, by the relationship

$$
\mathrm{N}_{\mathrm{D}}=\mathrm{N}_{\mathrm{t}} \mathrm{e}^{-\mu_{\text {atsp }}} \mathrm{e}^{-\mu_{m \mathrm{~m}}(\mathrm{t} t-1)}
$$

where $\mu_{\text {adip }}$ is the adipose tissue attenuation coefficient and $\mu_{\text {mix }}$ is the glandularadipose mixture attenuation coefficient. The assumption is that the beam is attenuated by $1 \mathrm{~cm}$ of adipose tissue (considering both of the outer adipose layers in the breast) and by $(\mathrm{t}-1) \mathrm{cm}$ of mixture tissue.

Then the glandular dose $D_{g}(x)$ at the depth $x$ is calculated to a small mass of glandular tissue embedded in a homogeneous medium with standard composition ( $50 \%$ adipose and $50 \%$ glandular tissue):

$$
\mathrm{D}_{\mathrm{g}}(\mathrm{x})=\mathrm{N}_{\mathrm{t}} \mathrm{E} \frac{\mu_{e n}^{\text {gland }}}{\rho^{\text {gland }}} \mathrm{e}^{-\mu_{\text {adip }} 0.5} \mathrm{e}^{-\mu_{\text {mix }}(\mathrm{x}-0.5)}
$$

where $\mu_{e n}^{\text {gland }}$ and $\rho^{\text {gland }}$ are the glandular tissue absorption coefficient and density, respectively.

The beam intensity at the depth $\mathrm{x}$ is attenuated by $0.5 \mathrm{~cm}$ adipose tissue and $(\mathrm{x}-$ $0.5) \mathrm{cm}$ mixture tissue. The mean glandular dose $\overline{\mathrm{D}}_{\mathrm{g}}$ is now evaluated integrating the glandular dose $\mathrm{D}_{\mathrm{g}}(\mathrm{x})$ over the breast mixed tissue region and dividing this number by the thickness:

$$
\bar{D}_{g}=\frac{1}{(t-1)} \int_{0.5}^{t-0.5} D_{g}(x) d x
$$




\section{Theoretical considerations}

In general the concept of "Image quality" can be considered to indicate the accuracy with which details can be perceived in an image. To avoid confusion about what this means it is necessary to define some terms and relationships which can lead to quantitative evaluation of the visibility of details in an image $(16,17)$.

A uniform photon fluence $\mathrm{N}_{\mathrm{i}}$ (photons per unit area) is incident on a phantom which has two adjacent regions. Region 1 consists of a thickness $t$ of a uniform material in the phantom without details whose total attenuation coefficient is $\mu(\mathrm{E})$. Region 2 is the region which contains the detail and it consists of a thickness ( $\left.t-t^{\prime}\right)$ of the same material plus a thickness $t$ ' of a material whose attenuation coefficient is $\mu^{\prime}(\mathrm{E})$. The transmitted photon fluences for the regions 1 and 2 are labeled $\mathrm{N}_{\mathrm{T} 1}$ and $\mathrm{N}_{\mathrm{T} 2}$, respectively.

The contrast $\mathrm{C}$ in an image can be defined as the difference in $\mathrm{x}$-ray transmission through region 1 and region 2 divided by the average of the two transmitted photon numbers:

$$
\mathrm{C}=\frac{\mathrm{N}_{\mathrm{T} 1}-\mathrm{N}_{\mathrm{T} 2}}{\left(\mathrm{~N}_{\mathrm{T} 1}+\mathrm{N}_{\mathrm{T} 2}\right) / 2}
$$

For a polychromatic beam we have to integrate over the energy and the contrast is:

$$
C=\frac{\int_{E} N_{T 1}(E) d E-\int_{E} N_{T 2}(E) d E}{\left(\int_{E} N_{T 1}(E) d E+\int_{E} N_{T 2}(E) d E\right) / 2}
$$

The transmitted x-ray fluences, $\mathrm{N}_{\mathrm{T1}}$ and $\mathrm{N}_{\mathrm{T} 2}$, consist of a primary (unscattered) component, $\mathrm{N}_{\mathrm{P} 1}$ and $\mathrm{N}_{\mathrm{P} 2}$, and a scattered component, $\mathrm{N}_{\mathrm{S} 1}$ and $\mathrm{N}_{\mathrm{S} 2}$, respectively such that

$$
\mathrm{N}_{\mathrm{T} 1}=\mathrm{N}_{\mathrm{P} 1}+\mathrm{N}_{\mathrm{S} 1} \text { and } \mathrm{N}_{\mathrm{T} 2}=\mathrm{N}_{\mathrm{P} 2}+\mathrm{N}_{\mathrm{S} 2}
$$

In the case of a monochromatic beam at a given energy the primary radiation is

$$
\mathrm{N}_{\mathrm{P} 1}=\mathrm{N}_{\mathrm{i}} \mathrm{e}^{-\mu t}
$$

and

$$
N_{p 2}=N_{i} e^{-\mu\left(t-t^{\prime}\right)} e^{-\mu^{\prime} t^{\prime}}
$$

Considering an equal contribution of scattered radiation that means $\mathrm{N}_{\mathrm{S} 1}=\mathrm{N}_{\mathrm{S} 2}=$ $\mathrm{N}_{\mathrm{S}}$, the contrast becomes

$$
\mathrm{C}=\frac{2\left(1-\mathrm{e}^{-\Delta}\right)}{1+\mathrm{e}^{-\Delta}+(2 \mathrm{~F} /(1-\mathrm{F}))}
$$


where $\Delta=t^{\prime}\left(\mu^{\prime}-\mu\right)$ and the factor $F$ is the scatter fraction defined as $F=N_{S} / N_{\mathrm{T} 1}$.

If the contribution of scattered radiation is uniform over the image, the number of scattered photons cancels in the numerator but it is present in the denominator, resulting in lower contrast. In the absence of scattered radiation the contrast depends only on the energy and on the absorption differences between the two materials (detail thickness, atomic number and density).

In the absence of scattered radiation and in the case of low contrast, $\mathrm{C}$ may be written

$$
\mathrm{C} \cong\left(\mu^{\prime}-\mu\right) \mathrm{t}^{\prime}
$$

In mammography the contrast is an important parameter because of the subtle differences in the transmission properties of the normal soft tissue and pathologic soft tissue masses and because of the importance of detecting minute details such as microcalcifications.

It is important to point out that radiographic contrast is actually influenced by two factors: the subject contrast and the receptor contrast. In the above considerations the concept of contrast refers to the subject contrast in which only the distribution of the photon intensity transmitted through the object is taken into consideration. We can suppose that the photons are counted by an ideal detector with efficiency $100 \%$. The receptor contrast takes into consideration the $x$-ray intensity pattern related to the image pattern detected by the detector. In this case, instead of the photon numbers $\mathrm{N}_{\mathrm{T} 1}$ and $\mathrm{N}_{\mathrm{T} 2}$, the corresponding output signal from the detector must be considered. For a film-screen system the $\mathrm{x}$-ray intensity pattern is related to the optical density pattern in the mammogram. The receptor contrast is affected by the film type and the processing conditions. For a digital detector with a simple linear relationship between the incident fluence $\mathrm{N}_{0}$ and the output signal $\mathrm{N}_{\mathrm{D}}=\mathrm{pf} \varepsilon \mathrm{N}_{0}$, the subject contrast and the receptor contrast are the same. ( $p$ is the pixel area, $f$ is the conversion factor of the signal and $\varepsilon$ is the detector efficiency). This relation holds only for monochromatic photon beams without scattered radiation since, in general, $f$ and $\varepsilon$ are energy dependent.

Image formation is a statistical process which involves the detection of a large number of photons. The inherent limitation to image information content is the statistical noise. The noise is generated in each component of an image system. It is possible to display all the image information content down to the appearance of "quantum mottle" which is the manifestation of the statistical noise

A parameter which takes into consideration the effect of the noise on the image quality is the signal-to-noise ratio (SNR). Considering the $\mathrm{SNR}_{\mathrm{in}}$ at the input to the detector (or considering an ideal detector with efficiency $100 \%$ ) for a detail with area $A$, and the photon numbers transmitted in regions 1 and $2\left(n_{T 1}=A N_{T 1}\right.$ and $\mathrm{n}_{\mathrm{T} 2}=\mathrm{AN}_{\mathrm{T} 2}$ ), one finds

$$
\mathrm{SNR}_{\mathrm{in}}=\frac{\mathrm{n}_{\mathrm{T} 1}-\mathrm{n}_{\mathrm{T} 2}}{\sqrt{\mathrm{n}_{\mathrm{T} 1}+\mathrm{n}_{\mathrm{T} 2}}}=\frac{\mathrm{C}}{2} \sqrt{\mathrm{n}_{\mathrm{T} 1}+\mathrm{n}_{\mathrm{T} 2}}
$$


The numbers of photons $\mathrm{n}_{\mathrm{T}}$ and $\mathrm{n}_{\mathrm{T} 2}$ vary according to a Poisson distribution with the noise equal to $\sqrt{\mathrm{n}_{\mathrm{T} 1}}$ and $\sqrt{\mathrm{n}_{\mathrm{T} 2}}$ respectively, which is the standard deviation of the photon number.

This equation shows that, unlike the contrast, the signal-to-noise ratio is dependent on the exposure as well as on the size of the detail because it is proportional to $\sqrt{\mathrm{AN}_{\mathrm{i}}}$. Scattered radiation, if it is able to enter the detector, produces a reduction of the $\mathrm{SNR}_{\text {in }}$.

The $\mathrm{SNR}_{\text {out }}$ of an image produced by a detector with a given Detective Quantum Efficiency (DQE) is related to the $\mathrm{SNR}_{\text {in }}$ as following

$$
\mathrm{SNR}_{\text {out }}=\sqrt{\mathrm{DQE}} \mathrm{SNR}_{\text {in }}
$$

\section{Analysis}

The acquired digital images have been processed by means of procedures created using the software "Interactive Data Language" (IDL) (18).

The images to be analyzed were acquired by means of an image plate detector (Section 3). This system is based on a photostimulable phosphor which can temporarily store an $\mathrm{x}$-ray image. The stored $\mathrm{x}$-ray pattern is subsequently read out by a scanning laser beam, which converts the trapped energy into photostimulated luminescence. The emitted luminescence, which is proportional to the absorbed $\mathrm{x}$-ray intensity, is detected by a photomultiplier whose output signal is logarithmically amplified and digitized with a 12-bit A/D converter. The gain of the logarithmic amplifier, which determines the range of the latitude, and the photomultiplier sensitivity (high voltage) are scaled according to the exposure level and the image contrast.

In the first phase of the analysis, the raw data, which are logarithmic values representing the $\mathrm{A} / \mathrm{D}$ converter, are linearized using the following formula:

$$
\text { data }_{\operatorname{lin}}=\left(\frac{\text { pixelsize }}{100}\right)^{2} * \frac{4000}{\mathrm{~S}} * 10^{\mathrm{L}\left(\frac{\mathrm{Raw}}{\mathrm{G}}-\frac{1}{2}\right)}
$$

where

$\mathrm{L}=$ Latitude (Dynamic Range; $1,2,3$ or 4 )

Raw $=$ raw data value $(0-255,0-1023$, or $0-4095)$

$\mathrm{G}=$ total gradation level $(256,1024$, or 4096$)$

$S=$ sensitivity $(400,1000,4000$, or 10000$)$

Pixel size $=100$ or 200 microns 
The parameters used in the June 1995 run were: $L=4, G=1024, S=4000$ and pixel size $=100$; in the August 1995 run they were: $L=4, G=1024, S=400$ and pixel size $=100$.

The image size was $85 \mathrm{~mm}$ (beam width) $\times 115 \mathrm{~mm}$ (translation length) in the June run, while it was $85 \mathrm{~mm}$ (beam width) $\times 95 \mathrm{~mm}$ (translation length) in the August run. Considering the pixel size of 100 microns, the number of image plate pixels per image was $850 \times 1150$ in the June experiment and $850 \times 950$ in the August experiment.

The phantoms used during the two experimental runs were the following: the ACR phantom (American College of Radiology), the CD phantom (Contrast-Detail), an anthropomorphic phantom (which simulates the breast tissue structures) and an excised breast tissue (the tumor was removed). Details of the ACR and CD phantoms are given in Figure 2.

The ACR phantom (Gammex: Model RMI 156) (Fig.2) is designed to attenuate $\mathrm{x}$-ray beams in the same way as a human breast of $50 \%$ adipose and $50 \%$ glandular tissue compressed down to a thickness of 40 to $45 \mathrm{~mm}$. Test objects of different sizes, shapes and densities are embedded in an insert. These test objects represent simulated malignancies such as micro-calcifications, fibrils and masses.

The CD phantom (Fig.2) is designed to evaluate the visibility limits of low contrast details of different thicknesses and diameter. It consists of a $15 \mathrm{~mm}$ thick Lucite background with circular areas of additional thickness. The circles are from $0.062 \mathrm{~mm}$ to $1 \mathrm{~mm}$ thick and the circle diameters are from about 0.3 to $7 \mathrm{~mm}$. Thus, the two materials which determine the contrast are Lucite and air.

In the June experiment we imaged the $\mathrm{CD}, \mathrm{ACR}$ and anthropomorphic phantoms using the following energies: 17, 18, 19.3, 20, 22, $24 \mathrm{keV}$. In the August experiment we imaged the CD and ACR phantom and the excised tissue at 16, 17, 18, $19 \mathrm{keV}$. In addition, imaging was done for the excised tissue at 20 and $22 \mathrm{keV}$ and for the $\mathrm{CD}$ phantom at $24 \mathrm{keV}$.

The list in Table 1 summarizes the phantom images produced by the image plate in the June and August beamtime at the X27C beamline. It specifies the run number, the energy, the kind of phantom and the corresponding mean glandular dose normalized to a $45 \mathrm{~mm}$ thick breast.

The images of the CD and ACR phantoms have been processed in order to achieve a flat background cancelling artifacts due to horizontal and vertical nonuniformities in the incident beam intensity. The horizontal modulations are due essentially to a non-uniform transmission by the monochromator and the $\mathrm{Be}$ window, while the vertical modulations are due to periodic oscillations of the translation stage. In the August run the latter problem was nearly completely removed by changing the position of the image plate and phantom to a more stable configuration.

The CD phantom images have been used for contrast and SNR measurements for different detail thicknesses at different energies. The largest diameter details $(7 \mathrm{~mm})$ have been analysed. The concept of receptor contrast has been applied and this contrast has been compared with the theoretical subject contrast for a monochromatic beam without scattered radiation using the relationship in Section 5.

In order to calculate the contrast an IDL procedure has been implemented. Once an image is displayed on a screen the procedure draws a circle on same image. It is 
possible to drag the circle on the image and change its diameter using the mouse. The first circle allows the selection of the detail area; then, the average value as well as the standard deviation of the pixels included into the circle is calculated. A rectangular box then appears and with the same operation it is possible to select and calculate the average value in a background area close to the detail. The procedure stores the results on a file and calculates the contrast. The $\mathrm{SNR}_{\text {out }}$ is determined using the photon fluence on the detectors.

The contrast for the same phantom details has been measured in three images of the $C D$ phantom produced by a conventional mammographic $\mathrm{x}$-ray tube at the University of North Carolina using a conventional film-screen as a detector and then digitized. The images were taken for phantoms of 15, 45, $75 \mathrm{~mm}$ thickness using 24, 25 and $30 \mathrm{kVp}$, respectively. The mean glandular dose is normalized to an exposure of $8 \mathrm{mR}$ to the film.

Figure 3 shows the plot of the measured image plate contrast as a function of the detail thickness at $18 \mathrm{keV}$, along with the theoretical curve. In the same plot the contrast measurements for the digitized film are shown $(45 \mathrm{~mm}$ thick phantom and 25 $\mathrm{kVp}$ ). The measured monochromatic beam contrasts are in good agreement with the theoretical values, while the digitized film values are lower than theoretical values. The comparison between experimental and theoretical data for $17,19.3,20,22,24 \mathrm{keV}$ is shown in Figure 4.

The plots of the measured contrast as a function of energy for three different detail thicknesses are compared with the theoretical curve in Figure 5. Good agreement is also obtained between the theory and experiment for the monochromatic data.

\section{Conclusions}

In two different periods of beamtime at the beamline $\mathrm{X} 27 \mathrm{C}$ af the National Synchrotron Light Source at Brookhaven National Laboratory, we have performed preliminary studies of mammographic imaging using a monochromatic synchrotron radiation source. We used both phantom objects and real tissue samples.

Qualitative studies with the contrast-detail phantom show good agreement when compared with the theoretical contrast. As expected, the contrast is higher if the energy is lower. The results show an improved contrast with energies $18 \mathrm{keV}$ and lower compared to images obtained from conventional polyenergetic $x$-ray imaging systems.

The results also show that for similar imaging conditions the monoenergetic mean glandular dose is less than that from polyenergetic sources. This is due both to the increased sensitivity of the image plate detectors and to actual reductions of dose for truely monochromatic beams. 


\section{Bibliography}

1. L.H. Baker, 'Breast cancer detection demonstration project: five-year summary report," CA 32(4) (1982) 194-225.

2. S.L. Saltzstein, 'Potential limits of physical examination and breast selfexamination in detecting small cancers of the breast: an unselected population-based study of 1302 cases," Cancer 54 (1984) 1443.

3. S. W. Fletcher, M.S. O'Malley and L.A. Bunce, 'Physicians' abilities to detect lumps in silicone breast models," JAMA 251 (1984) 1580.

4. P.C. Stomper and R.S. Gelman, 'Mammography in symptomatic and asymptomatic patients," Hem/Onc clinics NA, 3(4) (1989) 611-640.

5. X. Wu, E.L. Gingold, D.M. Tucker and G.T. Barnes, 'X-ray spectral effects on contrast and dose in mammography," Med. Phys. 21 (1994) 1006.

6. F. Carrol, J. Waters, W. Andrews, R. Price, D. Pickens, R. Wilhott, P. Tompkins, C. Roos, D. Page, G. Reed, A. Veda, R. Bain, P. Wang and M. Bassinger, "Attenuation of monochromatic x-rays by normal and abnormal breast tissues," Investigative Radiology 29 (1994) 266-272.

7. E. Burattini, M. Gambaccini, P.L. Indovina, M. Pocek and G. Simonetti, 'Synchrotron radiation: A new source in X-ray mammography,"Radiol. Med. 4 (1992) 181-188.

8. E. Burattini, E. Cossu, C. DiMaggio, M. Gambaccini, P. Indovina, M. Maryianai, M. Porek, S. Simeoni and G. Simonetti, "X-ray mammography with synchrotron radiation: A new high resolution technique valid for clinical application," Radiology 195 (1994) 239-244.

9. A. Mantykentta-Pramanick and R. Carr, "High Resolution mammography with Synchrotron Radiation," Medical Physics, submitted for publication (1995).

10. J.M. Boone and J.A. Seibert, "A comparison of mono- and poly-energetic x-ray beam performance for radiographic and fluoroscopic imaging," Med. Phys. 21 (1994) 1853-1863.

11. R.E. Johnston, D. Washburn, P. Pisano, W.C. Thomlinson, L.D. Chapman, N.F Gmur, Z. Zhong and D. Sayers, 'Preliminary experience with monoenergietic photon mammography," Medical Imaging 1995: Physics of Medical Imaging, SPIE 2432 (1995) 434-441. 
12. R.E. Johnston, D. Washburn, P. Pisano, C. Burns, W.C. Thomlinson, L.D. Chapman, F. Arfelli, N.F. Gmur, Z. Zhong and D. Sayers, 'Mammography phantom studies with synchrotron radiation," Radiology, submitted for publication.

13. 'Mammography: a user's guide." NCRP report 85. National Council on Radiation Protection and Measurements (Bethesda, Md, 1986).

14. B.J. Conway, ed. 'Nationwide evaluation of x-ray trends (NEXT), tabulation, and graphical summary of surveys, 1984 through 1987." Conference of Radiation Control Program Directors, CRCPD publication 89-3 (Frankfurt, KY, 1989).

15. G.R. Hammerstein, D.W. Miller, D.R. White, M.E. Masterson, H.Q. Woodard and J.S. Laughlin, "Absorbed radiation dose in mammography," Radiology 130 (1979) 485-491.

16. R.J. Jennings, R.J. Eastgate, M.P. Siedband and D.L. Ergun, "Optimal x-ray spectra for screen-film mammography," Med. Phys. 8 (1981) 629-639.

17. J.W. Motz and M. Danos, "Image information content and patient exposure," Med. Phys. 5 (1976) 8-22.

18. Research Systems, Inc., Boulder, Colorado 
Table 1a Run List (June 1995)

Run no. Filename Energy (keV) Phantom Average Glandular Dose(mrad)

\begin{tabular}{|c|c|c|c|c|}
\hline 1079 & $\mathrm{~cd} 1 . \mathrm{img}$ & 19.3 & $\mathrm{CD}$ & 67.9 \\
\hline 1096 & cd2_1096.img & 18 & $\mathrm{CD}$ & 89.2 \\
\hline 1118 & $\mathrm{~cd} 3 \_1118 \_17 \mathrm{kev} . \mathrm{img}$ & 17 & $\mathrm{CD}$ & 146.9 \\
\hline 1128 & cd4_1128_20real.img & 20 & $\mathrm{CD}$ & 48.5 \\
\hline 1143 & cd5_1143_22kev.img & 22 & $\mathrm{CD}$ & 27.8 \\
\hline 1155 & cd6_1155_24kev.img & 24 & $\mathrm{CD}$ & 20.4 \\
\hline 1201 & cd7_1201_18kev.img & 18 & $\mathrm{CD}$ & 7.0 \\
\hline 1203 & cd7_1203_18kev.img & 18 & $\mathrm{CD}$ & 21.3 \\
\hline 1205 & cd7_1205_18kev.img & 18 & $\mathrm{CD}$ & 28.4 \\
\hline 1207 & $\mathrm{~cd} 7$ 1207_18kev.img & 18 & $\mathrm{CD}$ & 45.5 \\
\hline 1209 & cd7_1209_18kev.img & 18 & $\mathrm{CD}$ & 51.2 \\
\hline 1211 & cd7_1211_18kev.img & 18 & $\mathrm{CD}$ & 64.1 \\
\hline 1213 & cd7_1213_18kev.img & 18 & $\mathrm{CD}$ & 71.0 \\
\hline 1215 & cd7_1215_18kev.img & 18 & $\mathrm{CD}$ & 99.7 \\
\hline 1217 & $\mathrm{~cd} 7$ 1217_18kev.img & 18 & $\mathrm{CD}$ & 121.2 \\
\hline 1090 & acrli.img & 19.3 & ACR & 40.1 \\
\hline 1109 & acr2_1109_18kev.img & 18 & $\mathrm{ACR}$ & 67.2 \\
\hline 1121 & acr3_1121_17kev.img & 17 & ACR & 137.5 \\
\hline 1130 & acr4_1130_20real.img & 20 & ACR & 46.6 \\
\hline 1149 & acr5_1149_22kev.img & 22 & ACR & 26.2 \\
\hline 1159 & acr6_1159_24kev.img & 24 & ACR & 17.0 \\
\hline 1080 & ant l.img & 19.3 & ANTR & 66.2 \\
\hline 1113 & ant2_1113_18kev.img & 18 & ANTR & 73.5 \\
\hline 1124 & ant3_1124_17kev.img & 17 & ANTR & 131.6 \\
\hline 1133 & ant4_1133_20real.img & 20 & ANTR & 48.5 \\
\hline 1151 & ant5_1151_22kev.img & 22 & ANTR & 25.8 \\
\hline 1162 & ant6_1162_24kev.img & 24 & ANTR & 14.2 \\
\hline
\end{tabular}


Table 1b Run List (August 1995)

Run no. Filename Energy (keV) Phantom Average Glandular Dose(mrad)

\begin{tabular}{|c|c|c|c|c|}
\hline 7019 & cd_7019.img & 16 & $\mathrm{CD}$ & 110.3 \\
\hline 7026 & cd_7026.img & 17 & $\mathrm{CD}$ & 68.6 \\
\hline 7029 & $\mathrm{~cd}^{-} 7029 . \mathrm{img}$ & 18 & $\mathrm{CD}$ & 48.5 \\
\hline 7032 & cd_7032.img & 19 & $\mathrm{CD}$ & 37.1 \\
\hline 7034 & cd_7034.img & 24 & $\mathrm{CD}$ & 18.6 \\
\hline 7014 & acr_7014.img & 16 & ACR & 105.4 \\
\hline 7011 & acr_7011.img & 17 & ACR & 65.6 \\
\hline 7008 & acr_7008.img & 18 & ACR & 45.9 \\
\hline 7003 & acr_7003.img & 19 & ACR & 34.9 \\
\hline 6010 & bio_6010.img & 18 & TISSUE & 96.8 \\
\hline 6011 & bio_6011.img & 18 & TISSUE & 57.9 \\
\hline 6012 & bio_6011.img & 18 & TISSUE & 155.3 \\
\hline 6023 & bio_6023.img & 16 & TISSUE & 328.2 \\
\hline 6026 & bio_6026.img & 17 & TISSUE & 164.6 \\
\hline 6031 & bio_6031.img & 19 & TISSUE & 64.4 \\
\hline 6036 & bio_6036.img & 20 & TISSUE & 37.3 \\
\hline 6041 & bio_6041.img & 22 & TISSUE & 23.1 \\
\hline 6106 & bio_6106.img & 16 & TISSUE & 106.9 \\
\hline 6109 & bio_6109.img & 17 & TISSUE & 66.6 \\
\hline 6112 & bio_6112.img & 18 & TISSUE & 47.1 \\
\hline 6115 & bio_6115.img & 19 & TISSUE & 35.1 \\
\hline
\end{tabular}




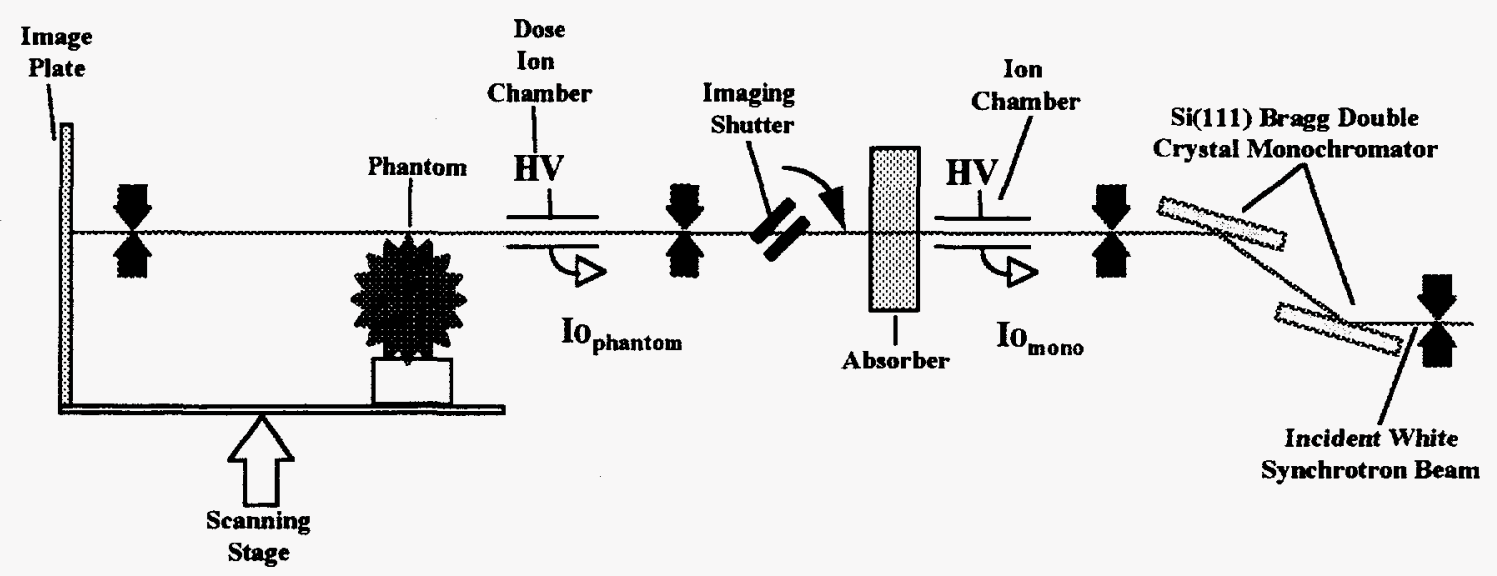

Figure 1: Mammogaphy imaging set-up (not to scale) 


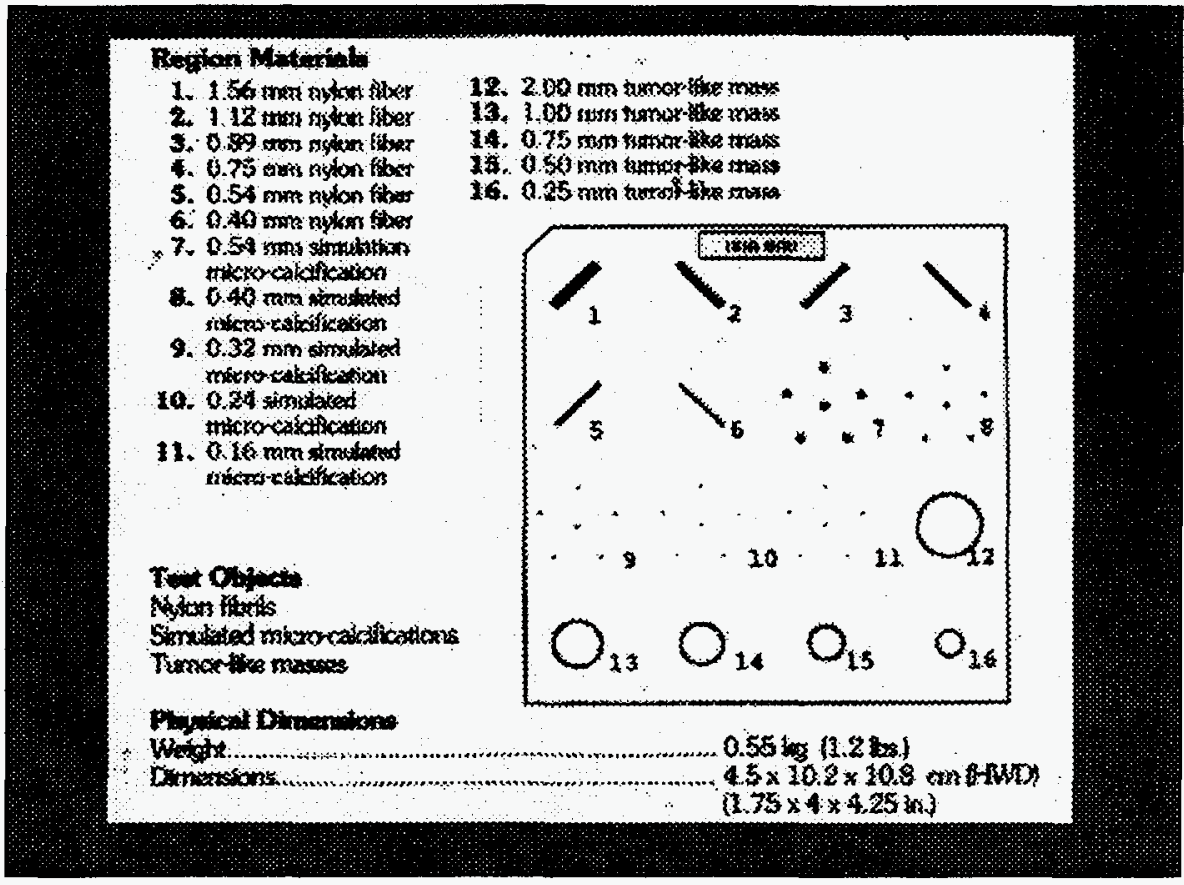

AMERICAN COLLEGE OF RADIOLOGY PHANTOM

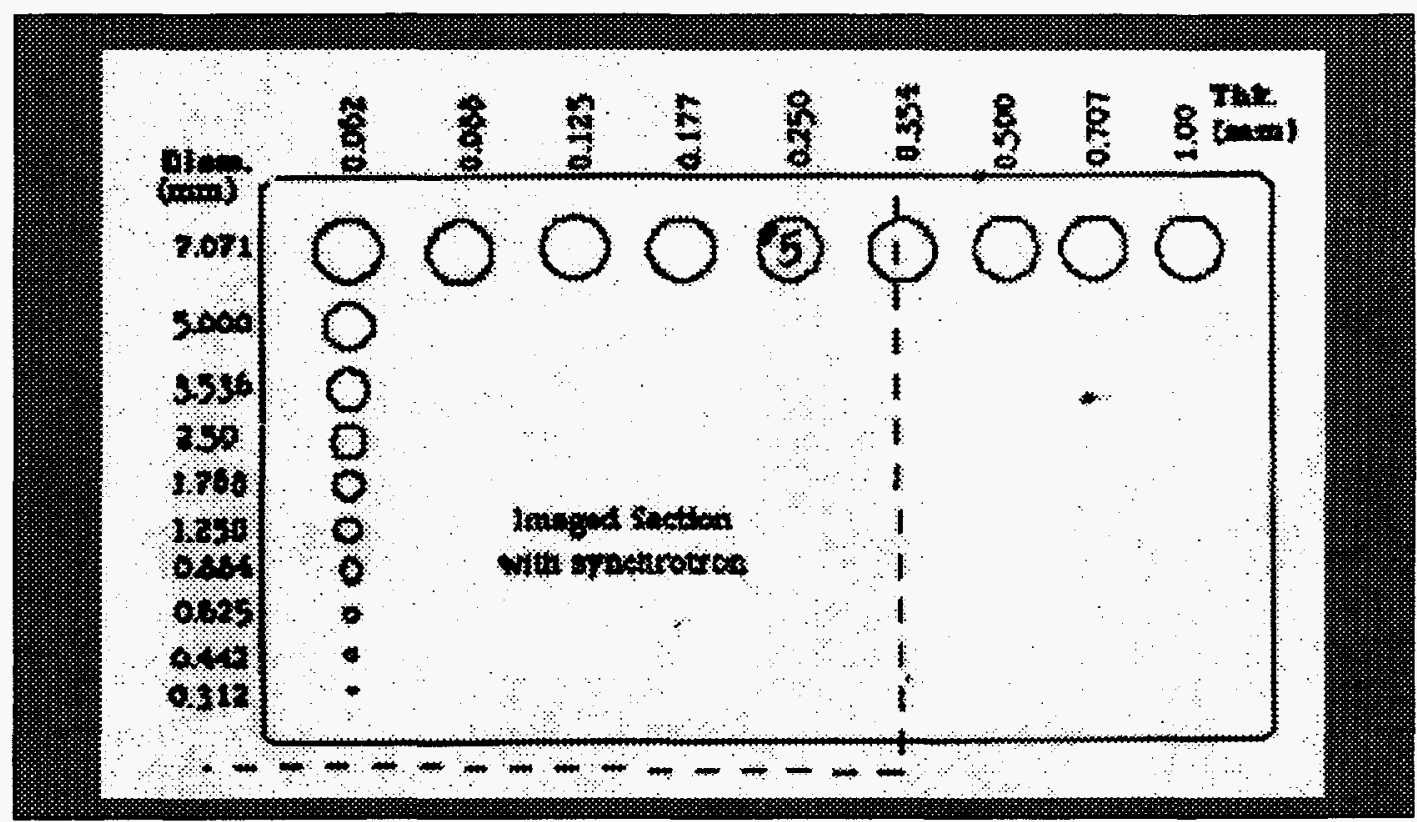

\section{CONTRAST DENSITY PHANTOM}

Figure 2: Details of the American College of Radiology Phantom and the Contrast Detail Phantom. 


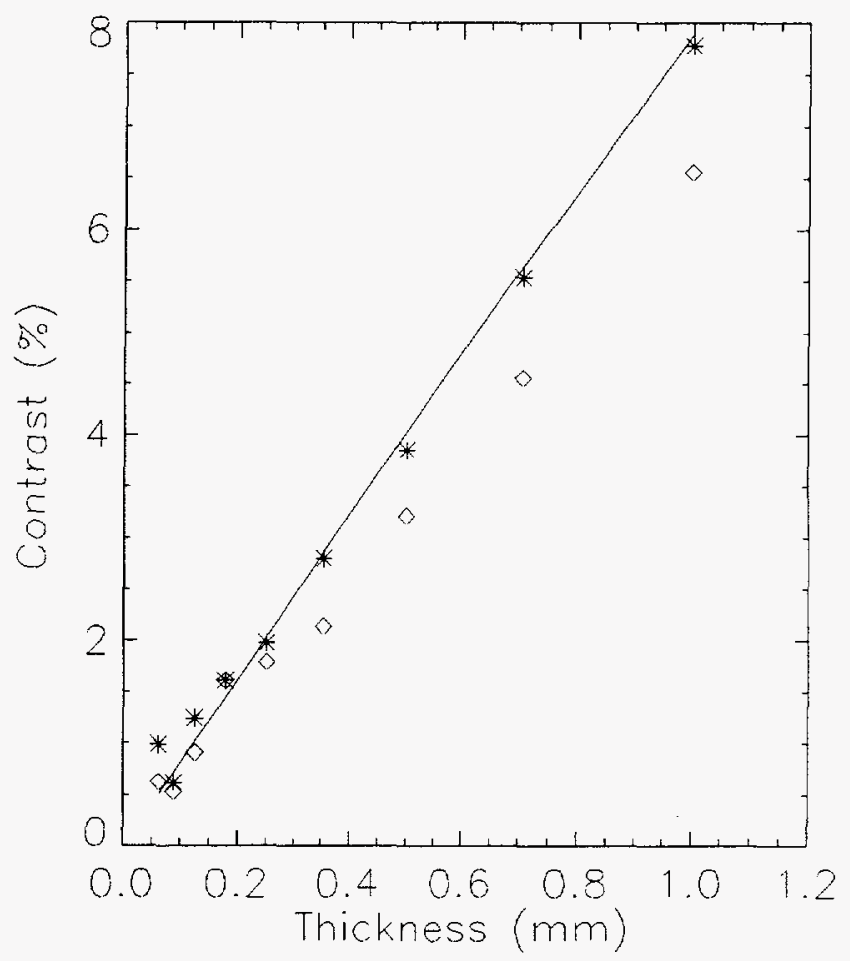

Figure 3: Measured contrast at $18 \mathrm{keV}\left({ }^{*}\right)$. The solid line represents the theoretical contrast. The measured contrast for a conventional film $(25 \mathrm{kVp})$ is also plotted $(\vartheta)$.

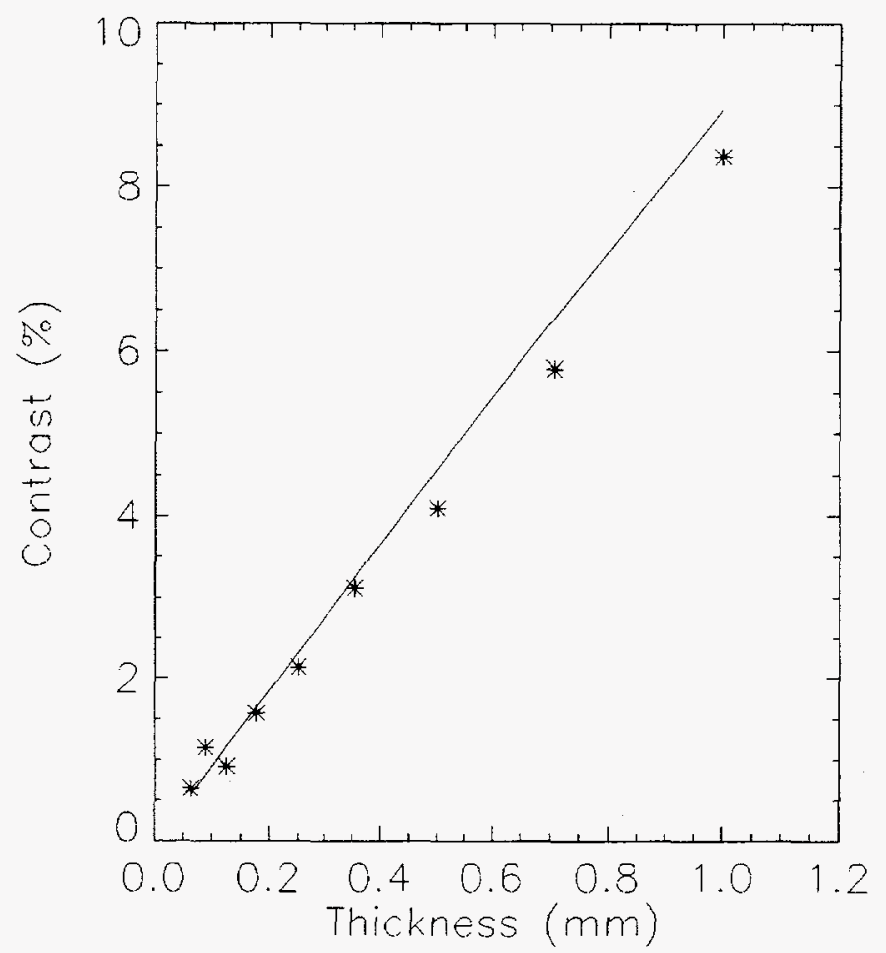

Figure 4a: Measured contrast at $17 \mathrm{keV}\left({ }^{*}\right)$. The solid line represents the theoretical contrast. 


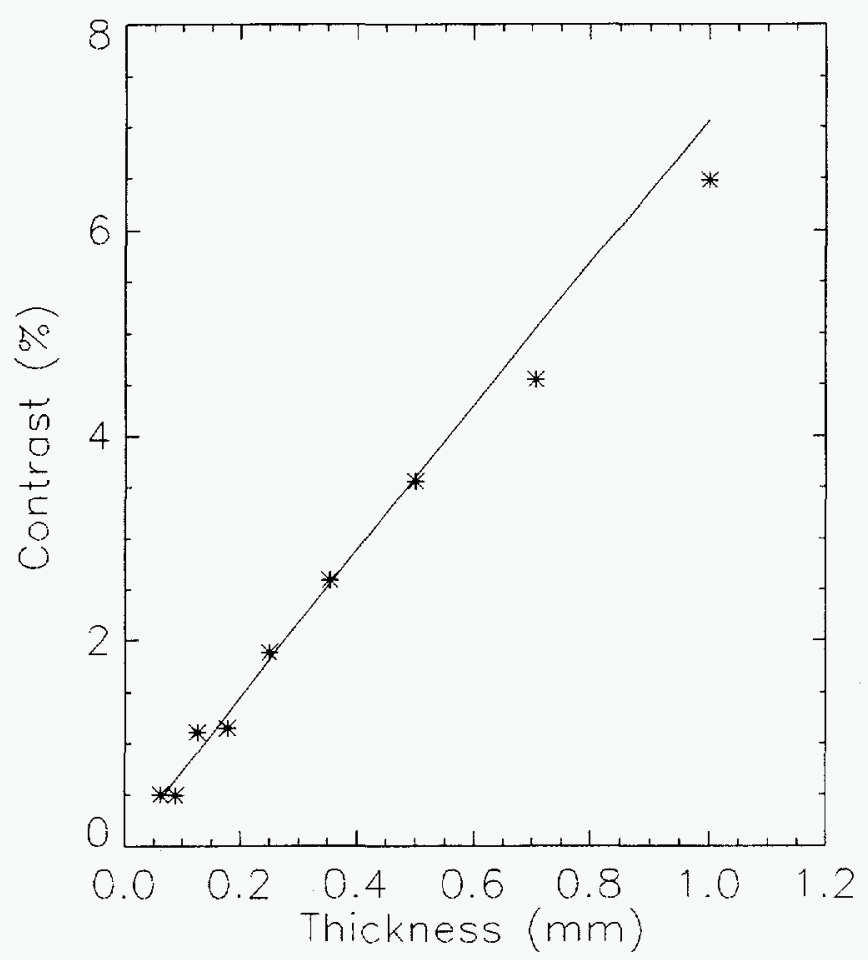

Figure $4 \mathrm{~b}$ : Measured contrast at $19.3 \mathrm{keV}\left({ }^{*}\right)$. The solid line represents the theoretical contrast.

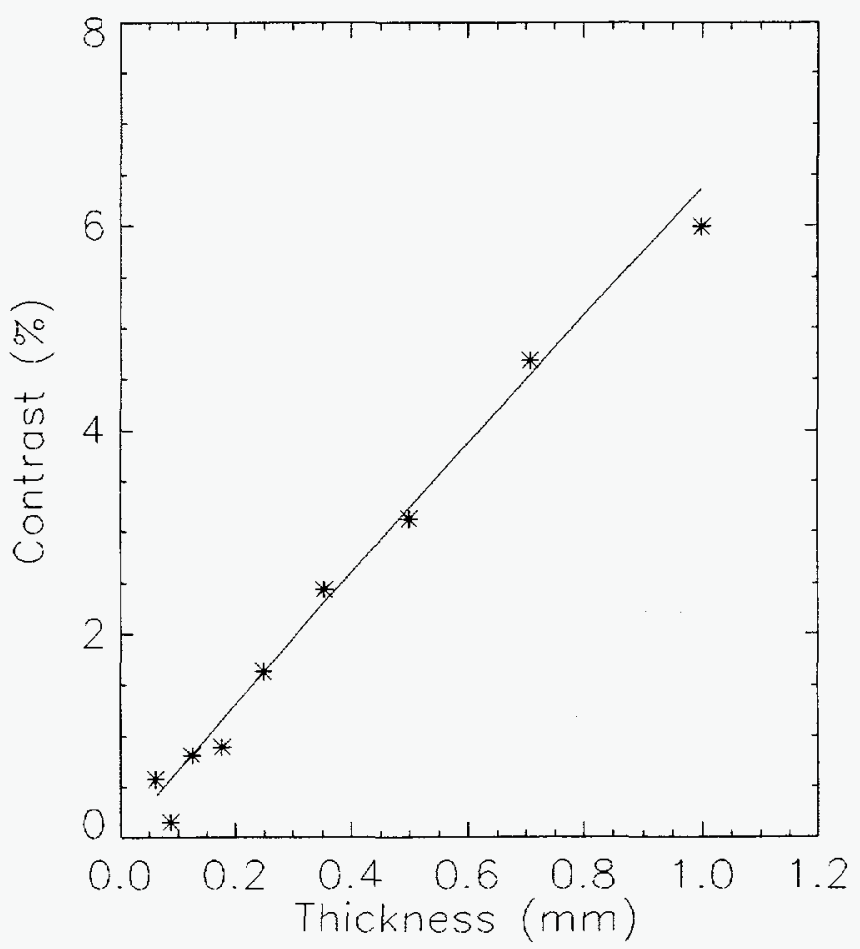

Figure 4c: Measured contrast at $20 \mathrm{keV}\left({ }^{*}\right)$. The solid line represents the theoretical contrast. 


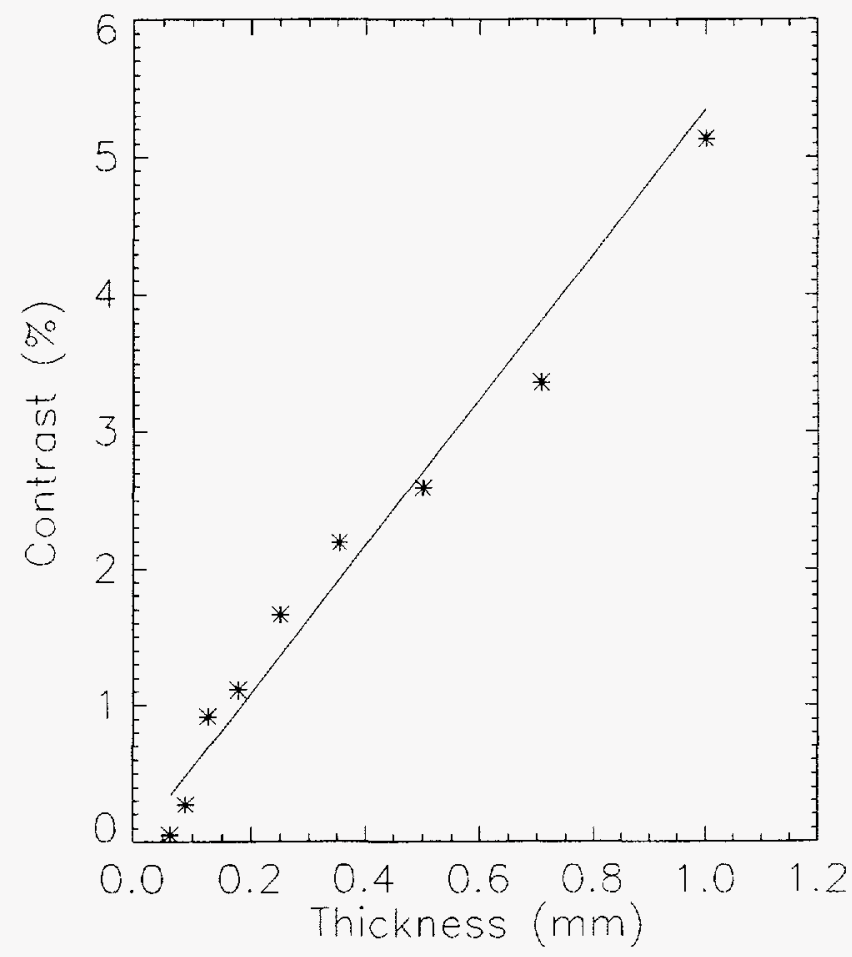

Figure 4d: Measured contrast at $22 \mathrm{keV}\left({ }^{*}\right)$. The solid line represents the theoretical contrast.

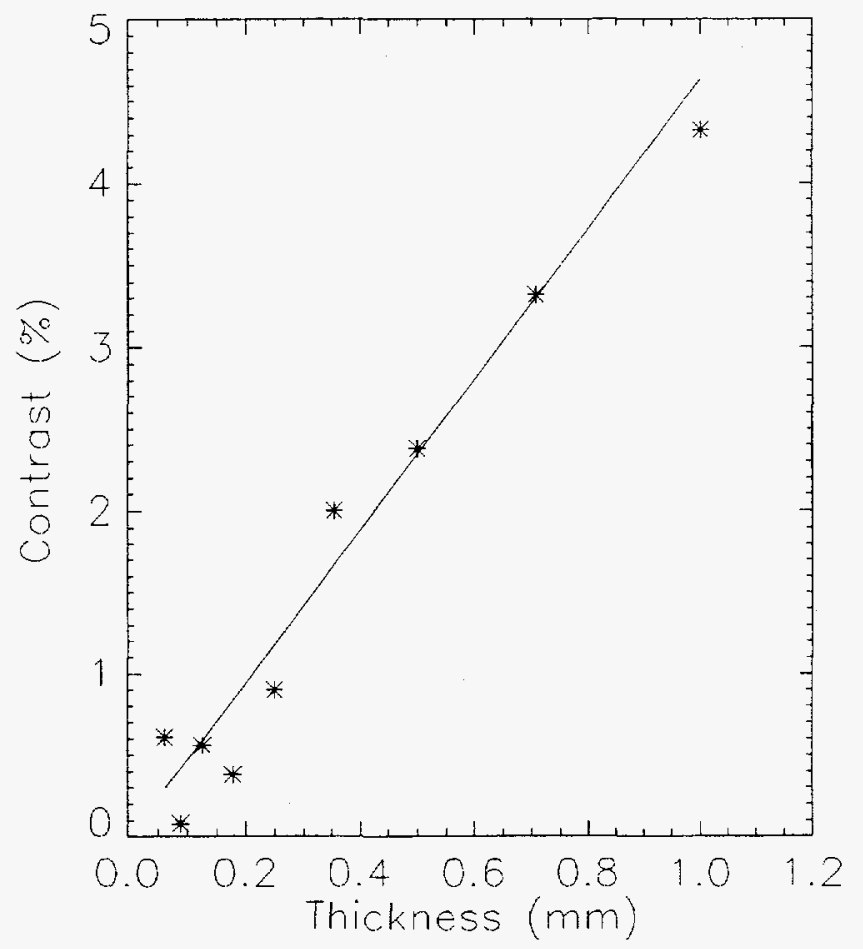

Figure 4e: Measured contrast at $24 \mathrm{keV}\left({ }^{*}\right)$. The solid line represents the theoretical contrast. 


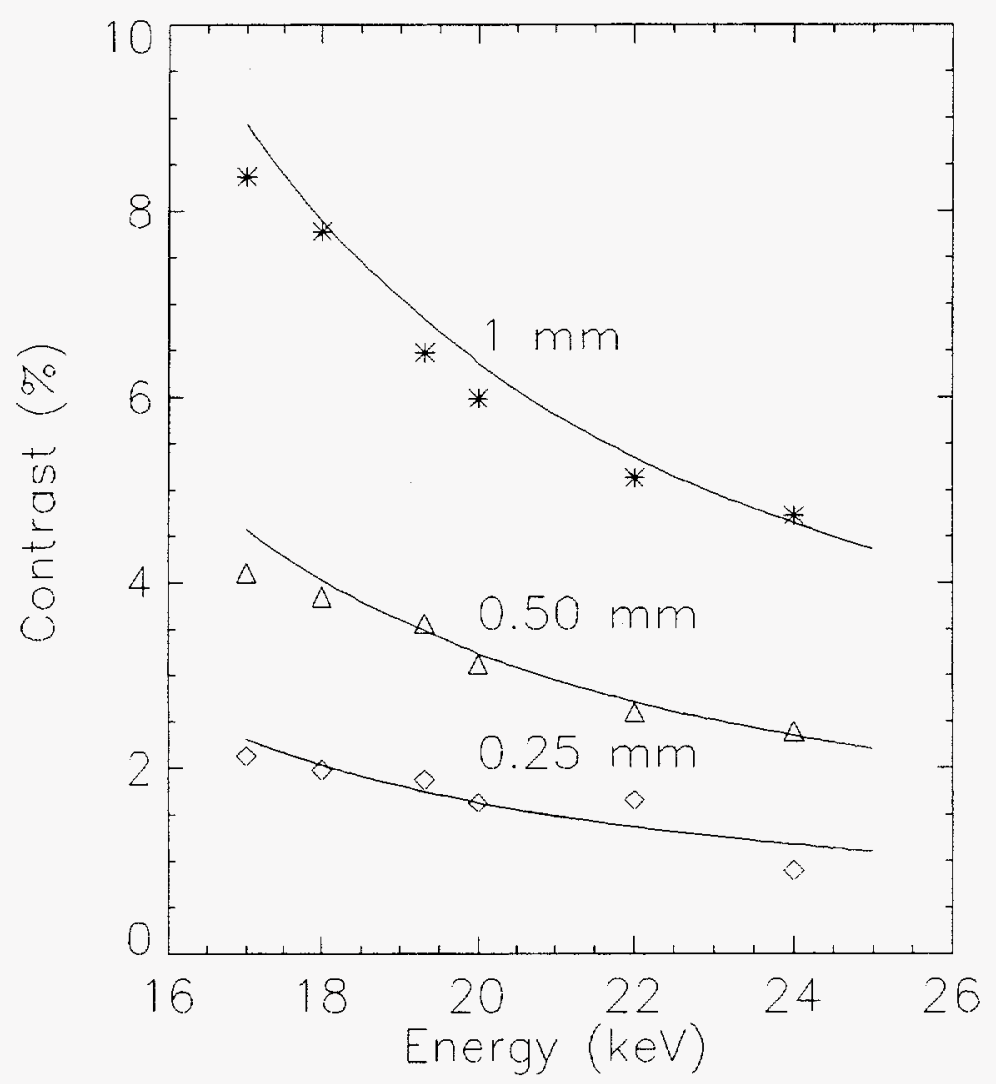

Figure 5: Measured contrast for different detail thicknesses. The solid line represents the theoretical contrast. 